\title{
Time course of encoding of patterns varying in array size and symmetry
}

\author{
EDMUND HOWE, JACK POWELL, KENNETH JUNG, and CYNTHIA BRANDAU \\ University of Missouri-St. Louis, St. Louis, Missouri
}

\begin{abstract}
The superior reproducibility of good, symmetrical patterns (the pattern goodness effect) is well documented. In the present experiment, the temporal course of the phenomenon was explored. Subjects attempted to recall 64 dot patterns having Array Sizes 4-7 and four levels of symmetry, following backward-masked exposure. It was found that all patterns were processed at the same rate, as is shown by a constant slope for all array size $\times$ symmetry $\times$ exposure time functions. An empirical index of pattern nameability was quite strongly correlated with symmetry and goodness. For Array Size 7 patterns, inclusion of the nameability index significantly raised the predictability of pattern recall above that observed with goodness value alone. These results suggest that when the immediate memory span is exceeded, the visual code may be supplemented by a verbal one.
\end{abstract}

If one looks briefly at an array of dots in a square matrix and then attempts to reproduce the pattern, performance will deteriorate not only as the number of elements increases beyond three or four, but also as the arrangement of elements becomes less symmetrical and the pattern poorer in the gestalt sense. This pattern goodness effect was predicted many years ago by gestalt psychologists (e.g., Hochberg, 1974; Koffka, 1935), and empirical evidence that good patterns are encoded faster than poor ones has been shown for 5-dot arrays (Bell \& Handel, 1976; Checkosky \& Whitlock, 1973; Howe \& Brandau, 1983), for 9-dot arrays (Garner \& Sutliff, 1974; Howe, 1980), and for other patterns as well (Attneave, 1955; Garner, 1974; Howe \& Jung, 1986).

In Howe and Jung's (1986) experiments, patterns consisting of 1-11 dots varying over four levels of symmetry were exposed for $1 \mathrm{sec}$. They found that (1) whole-pattern recall systematically declined as a function of array size and departure from symmetry, and (2) when floor and ceiling artifacts were controlled for, there was zero interaction between Array Sizes 4-8 and amount of symmetry.

The generality of these results raises the question of the temporal point in processing at which the pattern goodness effect might occur, and this question is addressed here. One theoretical possibility is that the symmetry advantage is a differential rate of encoding phenomenon and thus a time-bound monotonic function occurring over the first 300 or so milliseconds. A necessary empirical observation for this differential rate of encoding hypothesis

The research was supported in part by a grant from the University of Missouri Weldon Spring Fund. J. Powell is now at the University of Hartford, Connecticut, K. Jung is in the Market Research Department of Anheuser-Busch Companies, and C. Brandau is at Belleville College, Illinois. Send requests for reprints to Edmund Howe, Department of Psychology, University of Missouri-St. Louis, St. Louis, MO 63121. is that there should be a linear fan outcome, in this case a divergent interaction between amount of symmetry and available processing time. Such an observation would make rate of encoding dependent upon amount of symmetry. The absence of an interaction between symmetry and available processing time would suggest, on the other hand, that the symmetry advantage may entail an event occurring at a particular stage of processing.

The basic purpose of this experiment was thus to observe the time course of encoding of dot patterns varying in array size and amount of symmetry. Since the possibility obviously exists for recoding of visual patterns even following backward masking, an independent index of nameability for each pattern was also obtained.

\section{METHOD}

\section{Pattern Stimuli}

The 64 pattern stimuli consisted of dots set in a $5 \times 5$ grid, and were selected from a new set described in detail and presented with their empirical goodness values by Howe and Jung (1986). There were 16 patterns for each of the array sizes: 4, 5, 6, and 7 (AS4-AS7). Each set of 16 patterns consisted of four basic prototypes from which had been constructed three derivative patterns with decreasing amounts of whole and part symmetry. The four symmetry conditions will be conventionally identified by their rotation and reflection (R\&R) subset sizes as $R \& R(1-2), R \& R(4), R \& R\left(8_{H}\right)$, and $R \& R\left(8_{L}\right)$, where $H$ and $L$ refer, respectively, to high and low amounts of part symmetry (i.e., between quadrants) in $R \& R(8)$ patterns. A representative subset (25\%) of the patterns used and their goodness values appears in Figure 1.

\section{Pattern Nameability Values}

Ninety lower division students in introductory psychology classes at Belleville College served as subjects by attempting to generate a 1-2 word description of each pattern that they thought "would make it easier for someone to reproduce it from memory." Each pattern was presented for $5 \mathrm{sec}$, at orientation $0^{\circ}$ to 45 subjects, and at $90^{\circ}$ to the remaining ones.

For each orientation set, nameability was defined by the quantity $N / 45$, where $N$ refers to the number of subjects giving the same most common response. $N$ thus defines the strength of the primary name, and it is strongly correlated with the informational uncertainty statistic, $H$, 


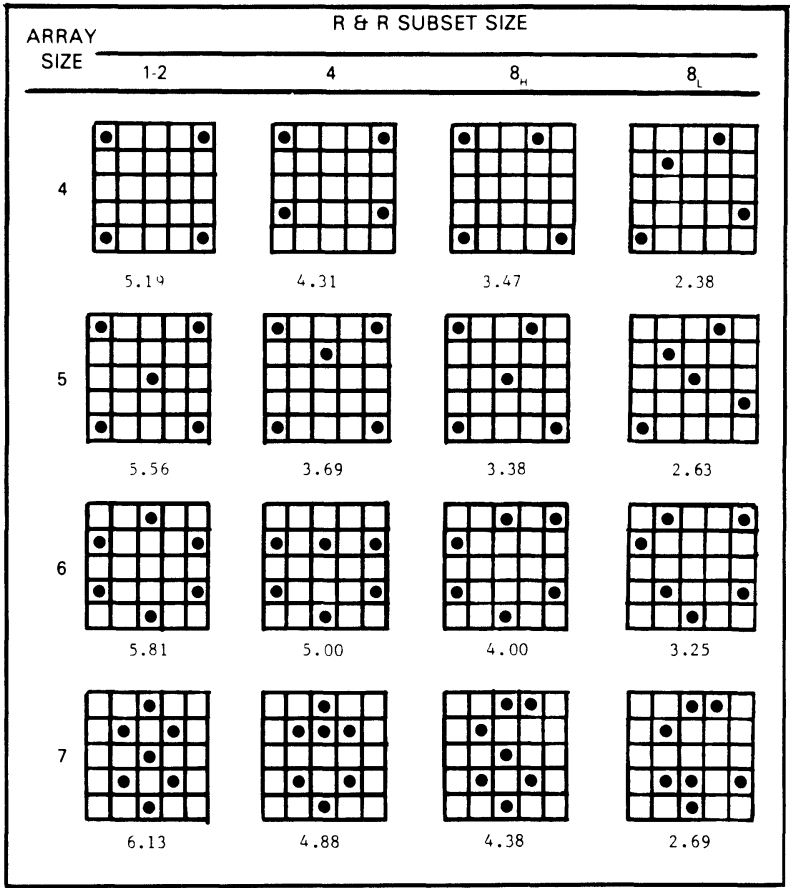

Figure 1. Representative sample of patterns from each array size and symmetry condition, and mean judged goodness values.

which in turn embraces the total distribution of free naming responses (Howe, 1972). Since the two sets of $N / 45$ values yielded within-arraysize $r$ s of $.92-.95$, they were pooled. Overall nameability values, which could range from 0.0 to 1.0, are shown in Table 1 .

\section{Apparatus}

Black-on-white target stimuli were viewed binocularly in a Model GB Scientific-Prototype 3-channel automatic tachistoscope, at an apparent viewing distance of $68 \mathrm{~cm}$. The projected pattern grid subtended an angle of $0.63^{\circ}$, each dot one of $0.07^{\circ}$. A fixation stimulus consisting of four L-shaped corners defined the exact spatial coordinates within which target patterns would appear. A backward mask consisting of a $5 \times 5$ grid with a dot in every cell appeared in the same visual-spatial position as the target stimulus.

\section{Procedure}

The fixation stimulus was exposed for $2 \mathrm{sec}$ and followed by a target for the designated exposure durations, which ranged overall from 80-380 msec for different array sizes (see Figure 2). The 100-msec mask onset was triggered by target offset. Subjects attempted to reproduce the target by drawing "dots or open circles" in the cells of a $4 \times 4 \mathrm{~cm}$

Table 1

Overall Mean Nameability Values, $N$, by Array Size and Amount of Symmetry

\begin{tabular}{cccccc}
\hline & \multicolumn{5}{c}{ Rotation and Reflection Subset Size } \\
\cline { 2 - 6 } Array Size & $1-2$ & 4 & $8_{\text {H }}$ & $8_{\mathrm{L}}$ & Mean \\
\hline 4 & .683 & .364 & .333 & .083 & .366 \\
5 & .320 & .192 & .167 & .072 & .188 \\
6 & .372 & .189 & .100 & .050 & .176 \\
7 & .483 & .156 & .156 & .037 & .208 \\
Mean & .463 & .225 & .188 & .060 & \\
\hline
\end{tabular}

Note-Subscripts $\mathrm{H}$ and $\mathrm{L}$ denote high and low amounts of part symmetry (between quadrants) in $R \& R(8)$ patterns. grid. Eight subjects were run in each condition. Four additional subjects were also run as controls in each AS condition, using an unmasked 500 -msec exposure of each target. The $32+16=48$ subjects were satisfying an introductory psychology course requirement at the University of Missouri-St. Louis.

\section{Design and Analysis}

Within each AS condition, the 16 target patterns were responded to four times, once in each of four rotational orientations (each orientation being confounded with a different one of four exposure times), by each subject. Targets, presentation orders, orientations, and exposure times were counterbalanced over subjects. The two dependent measures used were proportion of patterns out of four correctly recalled, and number of cells correct per pattern.

\section{RESULTS}

\section{Correct Pattern Recall}

Figure 2a-2d presents the data for correct pattern recall. Analysis of variance for each AS showed a main effect for amount of symmetry $\left[F \mathrm{~s}(3,21) \geq 19.01, M S_{\mathrm{e}} \leq 1.19\right.$, $p<.0001$ ], accounting for an average of $46 \%$ of the variance. A main effect for exposure time was significant for AS4-6 $\left[F_{\mathrm{s}}(3,21) \geq 5.96, M S_{\mathrm{e}} \leq 0.92, p=.005\right]$. For AS7, $F(3,21)=2.40, M S_{\mathrm{e}}=0.68, p>.10$, reflecting a marked floor effect for the $R \& R(8)$ patterns; and performance on those patterns barely improved under the unmasked 500-msec control condition. Despite local floor effects, the exposure time $\times$ symmetry interaction terms were below significance for all AS $[F(9,63) \leq 1.35$, $p>.20]$.

\section{Number of Cells Correct}

Figure $2 \mathrm{e}-2 \mathrm{f}$ summarizes the data using mean number of cells correct. For all AS, the main effects due to symmetry yielded $F_{\mathrm{s}}(3,21) \geq 24.87, M S_{\mathrm{e}} \leq 0.98, p<.0001$, and accounted for $35 \%$ of the variance. For exposure time $F_{\mathrm{S}}(3,21) \geq 5.87, M S_{\mathrm{e}} \leq 1.12, p \leq .005$. The exposure time $\times$ symmetry interaction terms showed $F \mathrm{~s} \leq 1.19$, $p>.30$.

\section{Array Size and Rate of Encoding}

The outcomes for all AS conditions were directly compared in a single analysis of variance for each dependent variable. For a test of the hypothesis that rate of pattern encoding was constant over array sizes, it is irrelevant that different AS conditions were accorded different absolute exposure times. In the following two analyses of variance, therefore, the within-AS exposure times were simply assigned arbitrary values of 1-4, and amount of symmetry and ordinal exposure time values were repeated factors, with AS independent.

The two AS main effects were significant. For patterns correct, $F(3,28)=6.05, p<.005$; and for mean cells correct, $F=15.69, p<.0001$. Critically, if rate of encoding did not differ as a function of AS, then the AS $\times$ exposure time and the AS $\times$ amount of symmetry $\times$ exposure time interactions should be negligible. For patterns correct, the interaction terms yielded $F(9,84)=$ 


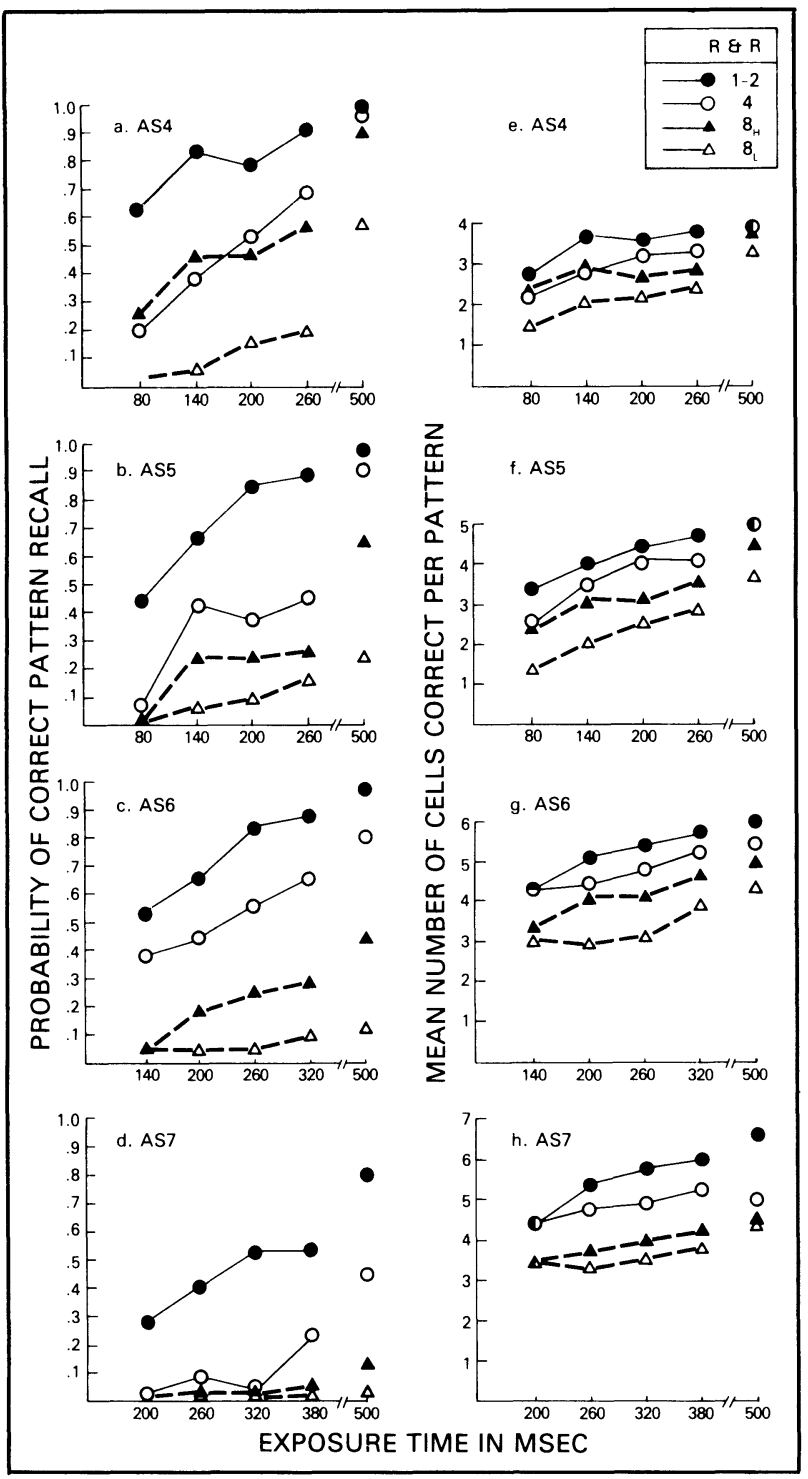

Figure 2. Mean recall values. (a)-(d): probability of correct pattern recall. (e)-(h): mean number of cells correctly recalled per pattern.

$1.11, p>.40$, and $F<1.0$, respectively. For the mean cells correct measure, both $F_{\mathrm{s}}<1.0$.

\section{Pattern Goodness and Pattern Recall}

Within AS conditions, the overall probability of correct recall was obtained for each of the 16 basic prototypical patterns (averaged over all orientations and exposure times). The four correlations, $r$, between those values and the mean pattern goodness values ranged from .82 to .89 (all $d f=14, p<.001$ ). Judged pattern goodness thus predicts individual pattern reproducibility quite well.

\section{Pattern Nameability and Pattern Recall}

Analysis of the data summarized in Table 1 shows that despite higher nameability of AS4 patterns $[F(3,12)=$ $9.63, p<.01]$, the AS $\times$ symmetry interaction was not significant $[F(9,36)=1.21, p>.30]$. However, symmetry accounted for over $80 \%$ of the nameability variance $[F(3,36)=28.80, p<.0001]$.

The within-array-size $r$ between nameability and recall were, respectively, $.86, .75, .87$, and $.89, d f=14$, $p<.005$. Nameability also is thus quite a good predictor of recall. The $r$ s between goodness and nameability were, respectively, .79, .84, .72, and .81 .

\section{Regression Analysis}

A stepwise regression analysis was run for each array size to establish whether either goodness or nameability significantly increased the variance accounted for by the other variable in the prediction of recall. For both AS4 and AS5, the $r^{2}$ between recall and goodness was .80 , and this proportion did not significantly increase when nameability was added in as a conjoint predictor. Inclusion of goodness as the second predictor, however, significantly raised the proportions of variance accounted for by nameability alone $\left(r^{2} \geq .51, R^{2} \geq .80, p \leq .002\right)$. For AS6 patterns, inclusion of either goodness or nameability as the second predictor raised the proportions of variance accounted for from .73 and .76 , respectively, to .86. Finally, for AS7, the value of $r^{2}$ when goodness alone was used as the sole predictor was .66; when just nameability was used, $r^{2}$ was .80 , and the value of $R^{2}$ was found to be $.82, p<.005$.

Thus, for AS4 and AS5, which are known to be at or close to the immediate memory span for nonsymmetric spatial locations (e.g., see Howe \& Jung, 1986; Sanders, 1968; Turvey, 1978), the availability of a verbal code appears not to enhance performance beyond the level produced by the visual encoding. For AS6, both codes appear to contribute equally to enhance recall performance. But for AS7 the availability of a verbal naming code appears to supplement the visual one.

\section{DISCUSSION}

Since the comparable slopes in the recall curves for all symmetry and array size conditions involve acceptance of the null hypothesis, it is most fitting to say that no evidence was found against the hypothesis of equal rates of encoding. While the empirical data do not provide a basis for assigning the symmetry advantage to a particular stage of processing, one matter merits brief comment.

It is suggested that pattern symmetry, like any other Lockean primary quality such as size, color, brightness, or movement, is a precategorical one that is abstracted before the exact configuration of the pattern. This idea, which is not novel (Fox, 1975; Howe, 1980; Locher \& Nodine, 1973), is reasonable on the grounds that any degree of symmetry can be defined by different configurations without involving any specific spatial locations. Several authors have discussed the reality of symmetry encoding mechanisms (e.g., Corballis \& Roldan, 1975; Julesz, 1971); others have adduced evidence that mechanisms for symmetry encoding may occur at an early level in the nervous system and are highly prefer- 
enced for the detection of different types of symmetry (Royer, 1978), and that detection of main axial symmetry is fastest for vertical, next fastest for horizontal, and slowest for other types of symmetry (Palmer \& Hemenway, 1978; Royer, 1981). Further, Howe (1980) found that judgments of pattern goodness were made at processing times far too short to permit recall from short-term visual memory.

The regression results implicate nameability for AS7 patterns, suggesting that, when the immediate visual memory span is exceeded, the visual code may be supplemented by a verbal one. But these considerations have no compelling bearing on the question of when and where such supplementary coding might take place. Pertinent in this regard is Marcel's (1983) demonstration that, following very brief backwardmasked exposures, semantic information was available to the subject even when visual information was clearly not available. Marcel's analysis displaces the assumption that representations governing early information processing are fundamentally identical to the representations experienced as conscious events. The verbal characterization is a case in point.

\section{REFERENCES}

ATtNeave, F. (1955). Symmetry, information, and memory for patterns. American Journal of Psychology, 68, 209-222.

BELL, H. H., \& HANDEL, S. (1976). The role of pattern goodness in the reproduction of backward masked patterns. Journal of Experimental Psychology: Human Perception \& Performance, 2, 139-150.

Checkosky, S. F., \& Whitlock, D. (1973). Effects of pattern goodness on recognition time in a memory search task. Journal of Experimental Psychology, 100, 341-348.

Corballis, M. C., \& Roldan, C. E. (1975). Detection of symmetry as a function of angular orientation. Journal of Expermental Psychology: Human Perception \& Performance, 1, 221-230.

Fox, J. (1975). The use of structural diagnostics in recognition. Journal of Experimental Psychology: Human Perception \& Performance, 104, 57-67.

GARNER, W. R. (1974). The processing of information and structure. Hillsdale, NJ: Erlbaum.

GARNER, W. R., \& SUTLIFF, D. (1974). The effect of goodness on encoding time in visual pattern discrimination. Perception \& Psychophysics, 16, 426-430.

Hochberg, J. (1974). Organization and the Gestalt tradition. In E. C.
Carterette \& M. Friedman (Eds.), Handbook of perception (Vol. 1, pp. 179-210). New York: Academic Press.

Howe, E. S. (1972). Number of different free associates: A general measure of associative meaningfulness. Journal of Verbal Learning \& Verbal Behavior, 11, 18-28.

Howe, E. S. (1980). Effects of partial symmetry, exposure time, and backward masking on judged goodness and reproduction of visual patterns. Quarterly Journal of Experimental Psychology, 32, 27-55.

HowE, E. S., \& BRANDAU, C.J. (1983). The temporal course of visual pattern encoding: Effects of pattern goodness. Quarterly Journal of Experimental Psychology, 61, 37-51.

HowE, E. S., \& JUNG, K. (1986). Immediate memory span for twodimensional spatial arrays: Effects of pattern symmetry and goodness. Acta Psychologica, 61, 37-51.

Julesz, B. (1971). Foundation of cyclopean perception. Chicago: University of Chicago Press.

KoffKa, K. (1935). Principles of Gestalt psychology. London: Routledge \& Kegan Paul.

LOCHER, P. J., \& Nodine, C. F. (1973). Influence of stimulus symmetry on visual scanning patterns. Perception \& Psychophysics, 13, 408-412.

MARCEL, A. (1983). Conscious and unconscious perception: Experiments on visual masking and word recognition. Cognitive Psychology, 15, 197-237.

Palmer, S. E., \& Hemenway, K. (1978). Orientation and symmetry: Effects of multiple, rotational, and near-symmetries. Journal of Experimental Psychology: Human Perception \& Performance, 4, 691-702.

ROYER, F. L. (1978). Computer generation of symmetric, semissymmetric, antisymmetric, and asymmetric patterns. Behavior Research Methods \& Instrumentation, 10, 170-176.

ROYER, F. L. (1981). Detection of symmetry. Journal of Experimental Psychology: Human Perception \& Performance, 7, 1186-1210.

SANDER, A. F. (1968). Short-term memory for spatial positions. Nederlands Tijdschrift voor psychologie, 23, 1-15.

TURVEY, M. T. (1978). Visual processing and short-term memory. In W. K. Estes (Ed.), Handbook of learning and cognitive processes (Vol. 5, pp. 91-142). Hillsdale, NJ: Erlbaum.

(Manuscript received April 24, 1989.) 